\title{
Polarization analysis of birefringence in uniaxially deformed silicon crystals
}

\author{
L.I. Berezhinsky ${ }^{1}$, I.L. Berezhinsky ${ }^{2}$, V.I. Pipa ${ }^{1}$, I.Ye. Matyash ${ }^{1}$, B.K. Serdega ${ }^{1}$ \\ ${ }^{I} V$. Lashkaryov Institute of Semiconductor Physics, NAS of Ukraine \\ 41, prospect Nauky, 03028 Kyiv, Ukraine \\ Phone: (38044) 525-5778, e-mail: serdega@isp.kiev.ua \\ ${ }^{2}$ I. Frantsevich Institute for Problems of Materials Science, NAS of Ukraine \\ 3, Krzhyzhanovsky str., 03142 Kyiv, Ukraine, e-mail: oleggrig@ipms.kiev.ua
}

\begin{abstract}
The birefringence induced by uniaxial compression in low-doped silicon crystals was investigated both theoretically and experimentally. The circular components of the Stokes vector in the transmission and reflectance radiation are measured as a function of external pressure by using the method based on the modulation of radiation polarization. The value of the Brewster constant was obtained for absorption $(\lambda=$ $0.63 \mu \mathrm{m})$ and transparence $(\lambda=1.15 \mu \mathrm{m})$ regions. The obtained experimental data are in a good agreement with calculation results based on the anisotropy model of dielectric properties within the framework of the Hook law. Shown is the practical importance of the polarization analysis in researching anisotropy of dielectric properties of materials.
\end{abstract}

Keywords: induced birefringence, polarization analysis, the Stokes vector.

Manuscript received 19.12.06; accepted for publication 26.03.07; published online 01.06.07.

\section{Introduction}

Birefringence in isotropic materials induced by uniaxial compression results in the polarization effect named as photo-elastic [1]. Its essence is that the refractive index value in the direction of compression and perpendicularly to it is different. In this case, the propagation of a light wave in the normal direction to the deformation axis with the polarization directed at some angle to this axis can be considered as propagation of two orthogonally polarized waves with different velocities. The phase difference between these waves is observed at the output from medium [2] and can be expressed by the formula

$\Delta \varphi=2 \pi\left(n_{\|}-n_{\perp}\right) L / \lambda$,

where $n_{\|}$and $n_{\perp}$ are the refractive indexes of material in parallel and perpendicular directions to the compression axis, respectively, $L$ is the thickness of medium, $\lambda$ is the light wavelength. The phase difference results in the fact that the linearly polarized light at the input into the medium becomes elliptically polarized at the output from it at any value of $\Delta \varphi$ excepting $\Delta \varphi=0$, $\pi / 2, \pi$. To determine the polarization state of radiation in this case, the Stokes vector is very convenient quantity.
The method of Stokes-polarimetry [3] is useful as components of the Stokes vector are directly measurable quantities. Stokes-vector components are especially easily measured by the method of modulation of radiation polarization.

The polarization modulation (PM) method for electromagnetic radiation was first used in ellipsometry [4]. In the work [5], the PM method was successfully used to study optical anisotropy in solids and liquids. The PM method allows to observe the photoelastic effect not only in transmittance, as it was done up to now, but also in reflection. Moreover, in [6] it was shown that this method allows to separate elliptically polarized radiation into the components of the Stokes vector (circular and linear polarized components). In this work, the photoelastic effect in crystalline silicon is analyzed using the Stokes vector components, which were experimentally measured by the PM method. It is shown that such description of the photoelastic effect allows to easily simulate the influence of the external factors (pressure, temperature etc.) on optical parameters of crystals, to deal with theoretical calculations and to compare them with experimental results.

The PM method allows register birefringence by two ways: (i) measuring the phase difference between orthogonal components of the linearly polarized 
radiation, and (ii) measuring the difference of reflection coefficients for $s$ - and $p$-polarized waves. In this work, we shall follow the (i)-way, and so we shall consider the anisotropy of the photoelastic effect as phase anisotropy contrary to amplitude anisotropy observed by the reflection absorption spectroscopy (RAS) method [7].

\section{Theory}

As it is known, the polarization state of a light wave in a general case can be described by the Stokes vector:

$S=[I, Q, U, V]$,

where $I$ is the total intensity of light, $Q$ is the intensity difference of light polarized across to axes, $U$ is the intensity of light polarized at the $45^{\circ}$ angle to the axes, and $V$ is the intensity difference of light with right and left circular polarizations. The Stokes parameters are expressed through the components $E_{x}=E_{\perp}$ and $E_{y}=E_{\|}$of the electrical field of a light wave as [8]

$I=E_{\|} E_{\|}^{*}+E_{\perp} E_{\perp}^{*}$,

$Q=E_{\|} E_{\|}^{*}-E_{\perp} E_{\perp}^{*}$,

$U=E_{\|} E_{\perp}^{*}+E_{\perp} E_{\|}^{*}$,

$V=i\left(E_{\|} E_{\perp}^{*}-E_{\perp} E_{\|}^{*}\right)$.

Let's consider a plate $(0 \leq z \leq L)$ of uniaxial crystal in vacuum, with optical axis oriented along $y$ axis. The permeability tensor of a crystal is defined by the components $\varepsilon_{x x}=\varepsilon_{z z} \equiv \varepsilon_{\perp}$ and $\varepsilon_{y y} \equiv \varepsilon_{\|}$. Let the light wave with the frequency $\omega$ and amplitude $\mathbf{E}=\left(E_{x}, E_{y}, 0\right)$ falls from vacuum in the direction of $z$ axis onto the surface $z=0$ of a sample. When refracting in the crystal, ordinary and extraordinary waves arise, which are characterized by complex refractive indexes, accordingly. The oscillations of electric fields of these waves are mutually orthogonal and independent from each other. Therefore, reflection (and transmission) of the waves with amplitudes $E_{i x}$ and $E_{i y}$ occur independently. It is possible to use for each wave the reflection $r_{v}$ and transmittance $t_{v}$ coefficients, respectively, obtained in [2] for an isotropic plate with the refraction indexes $\tilde{n}_{v}(v=\perp, \|)$ :

$r_{v}=\frac{r_{v 12}\left(1-e^{i \delta_{v}}\right)}{1-r^{2}{ }_{v 12} e^{i \delta_{v}}}, t_{v}=\frac{\left(1-r^{2} v 12\right)}{1-r^{2} v 12 e^{i \delta_{v}}} e^{i \delta_{v} / 2}$.

Here, $\delta_{v}=2 \omega L \tilde{n}_{v} / c$ and $r_{v 12}=\left(1-\tilde{n}_{v}\right) /\left(1+\tilde{n}_{v}\right)$ is the amplitude of reflection from a vacuum-crystal boundary. The vector amplitudes of the reflected and transmitted waves are expressed as

$\mathbf{E}_{r}=r_{\perp} E_{i x} \mathbf{e}_{x}+r_{\|} E_{i y} \mathbf{e}_{y}, \mathbf{E}_{t}=t_{\perp} E_{i x} \mathbf{e}_{x}+t_{\|} E_{i y} \mathbf{e}_{y}$.
The complex refractive indexes $\tilde{n}_{v}$ are expressed through real indexes of refraction $n_{v}$ and absorption $\chi_{v}$ as $\tilde{n}_{v}=n_{v}+i \chi_{v}$.

Under the uniaxial deformation of a crystal, the refractive index in the direction of pressure $\left(\tilde{n}_{\|}\right)$and in perpendicular (to it) direction $\left(\tilde{n}_{\perp}\right)$ are changed by different ways. As a model approach, we assume that the components of the permeability tensor have a linear dependence on the pressure $X$ :

$\varepsilon_{\|}=\varepsilon_{0}+a X, \quad \varepsilon_{\perp}=\varepsilon_{0}-a \mu X$.

Here, $\varepsilon_{0}=\left(n_{0}+i \chi_{0}\right)^{2}, n_{0}$ and $\chi_{0}$ are the indexes of refraction and absorption in the absence of pressure, $\mu$ is the Poisson constant, $a=a^{\prime}+i a^{\prime \prime}$ is the complex parameter depending on the frequency of light. For the weak absorption $\left(\chi_{0}<<n_{0}\right)$, from (5) in the linear approximation on $X$, we obtain

$n_{\|}=n_{0}+C_{1} X, \quad \chi_{\|}=\chi_{0}+C_{2} X$,

$n_{\perp}=n_{0}-\mu C_{1} X, \quad \chi_{\perp}=\chi_{0}-\mu C_{2} X$,

where $n_{\perp}, \chi_{\perp}$ and $n_{\|}, \chi_{\|}$are the indexes of refraction and absorption in directions perpendicular and parallel with respect to the direction of compression, respectively;

$C_{1}=a^{\prime} / 2 n_{0}, \quad C_{2}=\left(a^{\prime \prime} n_{0}-a^{\prime} \chi_{0}\right) / 2 n_{0}^{2}$.

Thus, in the case of absorbing crystal the dependence of its optical properties on uniaxial pressure is described by two parameters: $C_{1}$ and $C_{2}$. The Brewster constant $C=\left(n_{\|}-n_{\perp}\right) / X$ is expressed through $C_{1}$ as $C=(1+\mu) C_{1}$.

\section{Features of the experiment}

The circular component of the Stokes vector was measured in transmittance and reflection for Si crystal as a function of compressing effort. Measurements were carried out on the samples prepared from rather pure silicon single crystal with the concentration of impurities about $10^{13} \mathrm{~cm}^{-3}$. The type of crystal conductivity in our experiments was of no importance, as the contribution of electrons or holes to permeability, and hence to birefringence, is very small right up to the concentration of carriers about $\sim 10^{17} \mathrm{~cm}^{-3}$ [9]. In the course of measurements, light fell onto the $(010)$ plane of the sample. Uniaxial compression was applied along the crystallographic direction [100], which allowed to avoid transformation of a crystal into the biaxial one. To reduce down to a minimum the influence of superficial defects on results of measurements, the samples were treated using standard technology including chemicaldynamic polishing. The thickness of samples in the direction of light propagation was 0.3 and $1 \mathrm{~cm}$. Their cross section was $0.8 \times 1.0 \mathrm{~cm}^{2}$ that was stipulated by the type of the compressing device used. 
The optical scheme of the setup was performed as a Michelson interferometer and described in [5]. Its operation principle consists of the following. The linearly polarized radiation $\mathbf{E}=\left(E_{x}, E_{y}, 0\right)$ (azimuth $45^{\circ}$ relatively to the direction of pressure applied to the sample) falls on the splitter and is split by two beams with equal intensities. One of them is directed onto the anisotropic reflector $\mathrm{R}$ (quarter-wave plate) and another - on the sample normally to its surface. After reflection, both beams are reduced together and through the device analyzing polarization state are directed onto the photodiode. The block of the photoelastic modulator of polarization [4] and polarizer (Nicol prism) was used as an analyzing device. When the scheme operates in the "transmittance" mode the mirror is put behind the sample.

The feature of this scheme is use of a polarization modulator. The latter is a dynamic phase plate that changes polarization of a transmitted light wave without changing its intensity. The action of the system of modulator-polarizer has been analyzed in the work [6] in detail. It was shown that, after passing through the modulator and connected with it polarizer (Nicol prism), radiation with elliptic polarization generates in the photodiode a signal containing two components that differ by their frequencies. The first component with the modulation frequency $f$ corresponds to the intensity of a circular component of elliptically polarized radiation, and with the frequency $2 f$ - to a linearly polarized component. Therefore, it is possible to fix the intensity of a circular or linear component simultaneously by using the appropriate selective recording device. It is very important to note that in this case the magnitude of the linearly polarized component is the sum of $Q$ and $U$ (two components of the Stokes vector) which are orientated at the angle of $\pm 45^{\circ}$ relatively to each other. So, it is possible to measure the $Q$ and $U$ components by registration of an azimuth and magnitude of $I$ component of the Stokes vector.

All these measurements were carried out at the room temperature. The He-Ne laser ЛГ-126 $(\lambda=$ $0.63 \mu \mathrm{m}$ and $1.15 \mu \mathrm{m}$ ) was used as a source of radiation. The pure Si crystals weakly absorb at the wavelength $1.15 \mu \mathrm{m}$ (absorption coefficient is close to $10^{-1} \mathrm{~cm}^{-1}$ ) and strongly absorb at the wavelength $0.63 \mu \mathrm{m}$ (absorption coefficient is $3 \cdot 10^{3} \mathrm{~cm}^{-1}$ ) [10]. The Ge photodiode was used as a photodetector, signal of which was registered using a lock-in selective amplifier.

\section{Results and discussion}

Fig. 1 shows the comparison of calculated and experimentally observed circular component $V_{t}$ of the Stokes vector for the thick sample $(L=1 \mathrm{~cm})$ with weak light absorption. To estimate the parameters $C_{1}$ and $C_{2}$, in the function $V_{t}(X)$, we can neglect the weak dependence of reflection amplitudes $r_{v 12}$ on pressure. So, we can write

$$
V_{t}(X)=V_{0} \sin \left(\frac{\delta_{\|}-\delta_{\perp}}{2}\right) \exp \left(-\frac{2 \pi L}{\lambda}\left(\chi_{\|}+\chi_{\perp}\right)\right),
$$

where $V_{0}$ practically does not depend on pressure and $\left(\delta_{\|}-\delta_{\perp}\right) / 2=\varphi$. The cross-points of the function $V_{t}(X)$ with the $x$-axis correspond to the pressure $X_{m}$, at which the phase difference $\Delta \varphi=m \pi(m=0,1,2, \ldots)$. For $m=1$, the Brewster constant is expressed through the pressure $X_{1}$ as $C=\lambda /\left(2 L X_{1}\right)$. The constant $C_{2}$ defines a reduction of oscillation amplitudes with an increase of pressure.

The constant $C_{2}=1.3 \cdot 10^{-7}$ bar $^{-1}$ was determined from the experimental curve in Fig. 1 taking into account the attenuation of enveloping curve drawn around the oscillation function. The Brewster constant $C=$ $=2.6 \cdot 10^{-6}$ bar $^{-1}$ (appropriate constant $C_{1}=2 \cdot 10^{-6}$ bar $^{-1}$ ) was determined from the same experimental curve in the point $X_{1}$, in which the amplitude of a signal is equal to zero, i.e. $V_{t}=0$, and the phase difference $\left(\delta_{\|}-\delta_{\perp}\right)=$ $=\pi(m=1)$.

The damping character of intensity of circular polarized component is explained by an increase of the absorption coefficient $\chi_{\|}$and decrease of the absorption coefficient $\chi_{\perp}$ with growing the pressure according to (6). In fact, it displays the action of birefringence and dichroism effects simultaneously.

Using expressions (3)-(5), we have calculated all the components of the Stokes vector. The Stokes vector components as the function of pressure in transmittance are shown in Fig. 2. Let's pay attention to the following fact: the total intensity weakly oscillates with a change

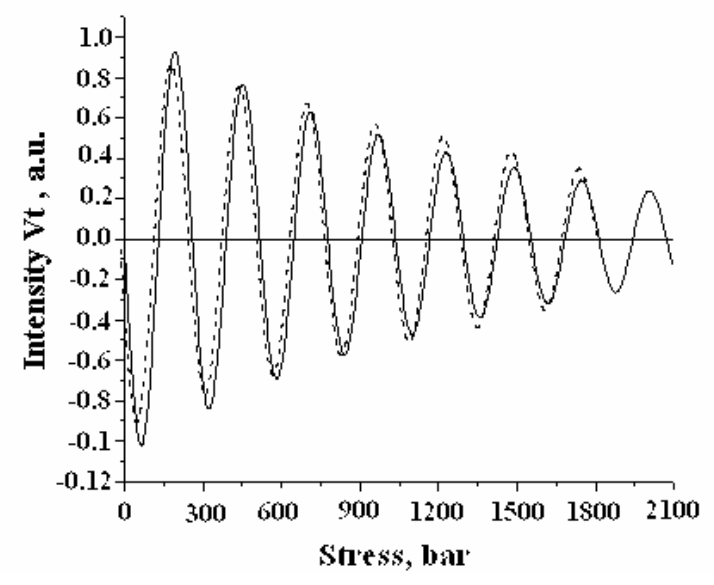

Fig. 1. $V_{t}$ component of transmitted light as a function of the uniaxial compression $X$ for the sample with the thickness $L=0.3 \mathrm{~cm}$ : dashed line - experiment, solid line calculation by using (3)-(5) for the values: $\omega=$ $1.6 \cdot 10^{15} \mathrm{~Hz} ; n_{0}=3.5 ; \chi_{0}=5 \cdot 10^{-5}$. 
of pressure. At the same time, the oscillation of the $I_{t}$ component is synchronous with oscillation of the linear component $Q_{t}$. These oscillations are due to multibeam interference that inevitably occurs when using the parameters of silicon. Our calculation showed that these oscillations disappear, if the absorption index increases up to $\chi_{0}=2 \cdot 10^{-4}$. The explanation of behaviour of the $U_{t}$ and $V_{t}$ components is not difficult, too. Let's remember that the $U_{t}$ component characterizes a linear polarization of the electric field of the light wave with the azimuths $\pm 45^{\circ}$ relatively to the deformation direction. The incident light wave has just this polarization. The sample takes the form of a phase plate under compression, and accordingly with its properties, it transforms the incident linear polarized wave into the elliptically polarized one in a general case. Fig. 2 illustrates this situation. One can see that the $V_{t}$ component grows at the expense of a decrease of the $U_{t}$ component when the pressure increases. At the argument $X=48$ bars, the $U_{t}$ component is zero and the $V_{t}$ component achieves its peak value. It means that the light wave leaving the sample is completely circular polarized because the phase difference $\varphi$ between orthogonal components of the linearly polarized radiation achieves $\pi / 2$. The further increase of the pressure results in appearance of the $U_{t}$ component again but with another sign. At the same time, the intensity of the $V_{t}$ component decreases. At $X=$ $=85$ bars, $V_{t}=0$ and the $U_{t}$ component has its peak value. In this point, the phase difference $\varphi=\pi$, and the light wave leaving the sample has linear polarization but orthogonally to polarization of the incident wave. The further increase in pressure after the point $X=85$ bars results in growth of the circular $V_{t}$ component and decrease of the $U_{t}$ component. However, the circular $V_{t}$ component has another sign that means changing of the rotation direction. The same situation is observed at the point $X=176$ bars and other appropriate points.

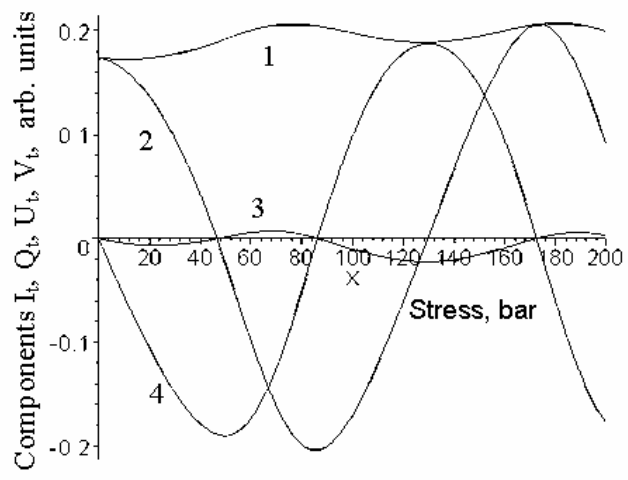

Fig. 2. Components of the Stokes vector for transmitted light as a function of the uniaxial compression $X$ calculated using (3)-(5): $1-I_{t}, 2-U_{t}, 3-Q_{t}, 4-V_{t}$ for the values: $\omega=1.6 \cdot 10^{15} \mathrm{~Hz}, n_{0}=$ $=3.5 ; L=0.3 \mathrm{~cm} ; \chi_{0}=5 \cdot 10^{-5} ; C=2.6 \cdot 10^{-6} \mathrm{bar}^{-1}$.
It is unexpected that $Q_{t}$ components of the Stokes vector appear, i.e. the linearly polarized wave with the azimuth parallel to the optical axis of the sample (direction of deformation), which is absent in the structure of initial radiation at $X=0$. The appearance of this component is undoubtedly connected with a combination of the $U_{t}$ and $V_{t}$ components, which takes place at the multiple reflection of radiation inside the sample. Appearance of the $Q_{t}$ component is a reverse process relatively to resolution of the elliptically polarized wave by linear and circular components, which are carried out by means of the used technique. It is confirmed by the fact that the $Q_{t}$ component is present when $V_{t} \neq 0$ and $Q_{t}=0$ at $V_{t}=0$ (excluding the first period). Moreover, the calculation showed and experiment confirmed that the $Q_{t}$ component disappears when the absorption coefficient or thickness of the sample increases, i.e. when multireflections are absent due to a complete damping of radiation along the sample.

Let's note the important feature of the PM method in measurements of the photoelastic effect. The nonpolarized component of a light flow is always present after passage through the modulator and polarizer, because these elements are not ideally perfect. Therefore, this component is a handicap in measurements of small anisotropy, because it will create a noise current in the photodiode. In the case of PM, this nonpolarized component does not contribute to the modulated signal and consequently does not limit the sensitivity of the used selective amplifier. Thus, the range of measured signals (and hence measured anisotropy) can reach some orders as is shown in Fig. 3. It shows the first half of the period of an oscillation function for the circular component of the Stokes vector submitted in Fig. 2. The curve in Fig. 2 is bounded below by a noise of the measuring circuit and light source.

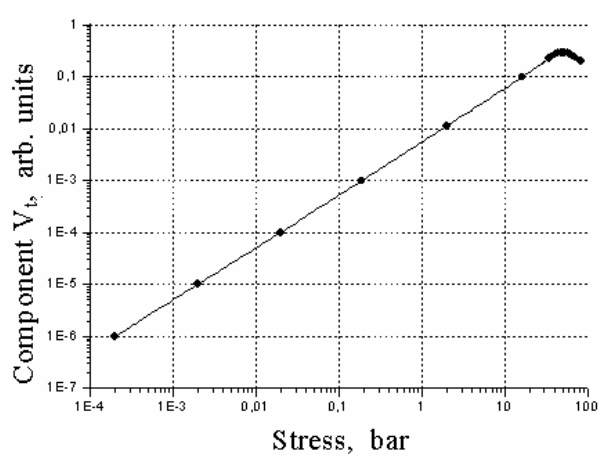

Fig. 3. Experimental illustration for detectability of PM in relation to the value of compression obtained from the first half-period of the $V_{t}$ oscillation function in Fig. 2. 
The investigations of induced birefringence in silicon was carried out earlier only in transmittance by means of the traditional optical-polarization schemes. So, it is interesting to compare the magnitude of this constant obtained earlier, for example in the work [11], where $C=1.95 \cdot 10^{-6} \mathrm{bar}^{-1}$, and that of our PM method. The value of the Brewster constant at $\lambda=1.15 \mu \mathrm{m}$ determined in our experiments is $C=2.6 \cdot 10^{-6} \mathrm{bar}^{-1}$. Distinction with the result obtained in the work [11] is close to $30 \%$.

The measurement of the photoelastic effect in reflection was carried out at $\lambda=1.15 \mu \mathrm{m}$ (case of weak absorption) and $\lambda=0.63 \mu \mathrm{m}$ (case of strong absorption). The effect of multireflection with participation of the sample back wall plays an important role in formation of the reflected wave in the first case, but is insignificant in the second case. Fig. 4 shows the results of calculations of the $I_{r}, U_{r}, Q_{r}, V_{r}$ components as a function of the pressure $X$ for the case of weak absorption. As well as in

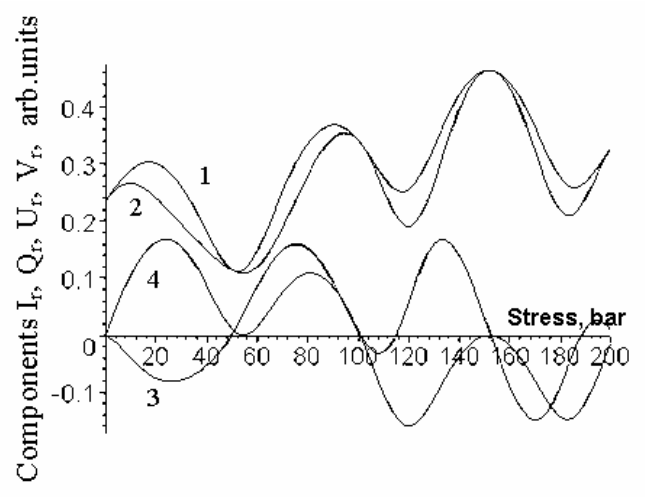

Fig. 4. Components of the Stokes vector $\left(1-I_{r}, 2-U_{r}, 3-\right.$ $\left.Q_{r}, 4-V_{r}\right)$ as a function of the uniaxial compression $X$ calculated using (3)-(5) for radiation reflected from the sample for the values: $L=0.3 \mathrm{~cm} ; \omega=1.6 \cdot 10^{15} \mathrm{~Hz}$; $n_{0}=3.5 ; \chi_{0}=5 \cdot 10^{-5} ; C=2.6 \cdot 10^{-6}$ bar $^{-1}$.

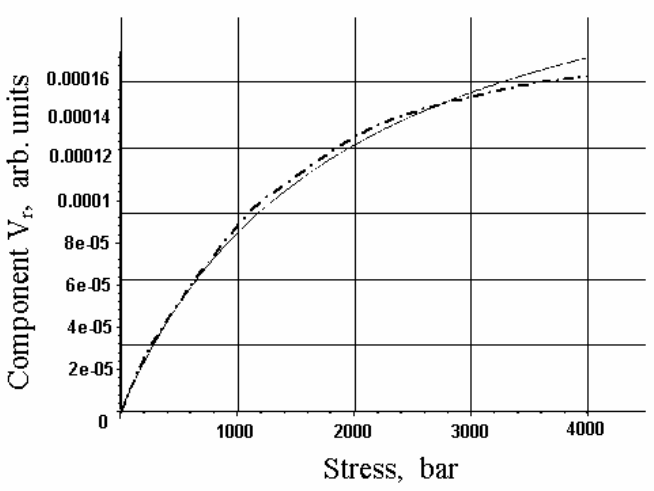

Fig. 5. $V_{r}$ component of the Stokes vector as a function of the uniaxial compression $X$ : dashed line - experiment, solid line - calculation by using (3)-(5) for the values: $L=$ $=0.3 \mathrm{~cm} ; \omega=3 \cdot 10^{15} \mathrm{~Hz} ; n_{0}=3.8 ; \chi_{0}=3.5 \cdot 10^{-3} ; \quad C=$ $=4 \cdot 10^{-6} \mathrm{bar}^{-1}$. transmittance, multibeam interference takes place in this case, and then all the components $I_{r}, U_{r}, Q_{r}, V_{r}$ are the oscillation functions versus $X$. Notice that the optical path is increased twice at participation of a back wall in formation of the reflected wave. It means that, within the framework of the accepted model (6), in the same range of pressures the oscillation period for the reflected wave should decrease twice in comparison with the transmitted wave, as is observed in Fig. 4.

The curve $R=f(X)$ of the photoelastic effect in the case of strong light absorption (Fig. 5) essentially differs from that of the transmittance case. This difference is observed both in the shape and in absolute value. First of all, it is caused by the fact that the crystal thickness $d$ forming a reflected wave $\left(d=1 / \alpha \approx 3.3 \cdot 10^{-4} \mathrm{~cm}\right.$ for $\lambda=$ $0.63 \mu \mathrm{m})$ is some orders less than the geometric crystal thickness of $0.3 \mathrm{~mm}$. Hence, in this case, the phase difference $\varphi=(2 \pi \lambda) \Delta(n L)$ between orthogonal components of a light wave should be $L / 2 d$ times less than in the transmittance case at the same anisotropy of $\Delta n$. The real situation is still more complex. For example, Fig. 5 shows the calculated and experimental curves of the $V_{r}$ component of the Stokes vector. The analysis of the experimental curve has shown that this curve is not described by a sine function, as it is observed in transmittance, but rather it is some power function. Moreover, the value of the Brewster constant, at which the best coincidence of the experimental and calculated curves takes place, also differs from the meaning of that in the transparency region. This result is in a good agreement with those of the work [12], where by means of the Raman scattering in silicon shown is that the Brewster constant sharply grows when approaching to the absorption edge.

\section{Conclusions}

The use of polarization analysis to study birefringence shows that the characteristics and peculiarities of the photoelastic effect are well displayed by two components of the Stokes vector, namely, $U_{t}$ and $V_{t}$. However, the use of the circular component $V_{t}$ for measurements of any specific quantities (for example, the Brewster constant or anisotropy value) represents the most optimum variant. It is explained by the fact that the function $V_{t}(X)$ has linear dependence on the birefringence value, at least, for small meanings of the argument, and it can be measured within a wide range of signals. It is advantages of the $V_{t}(X)$ function. As signals measured by standard devices have a noise level of 5-6 orders less than the maximal value of the $V_{t}$ component, the sensitivity of the measuring PM-devices to the quantity of anisotropy is some orders higher than traditional optical-polarization methods. So, investigating anisotropy of refraction indexes is enough to be limited by such value of the product $\left(n_{\|}-n_{\perp}\right) L$, until the function $V_{t}=f(X)$ has linear dependence, i.e. $\varphi<\pi / 2$. 
Concerning to reflection, the results obtained by means of the PM method testify that this method is very promising to investigate birefringence induced in opaque substances. The nonlinearity observed in the deformation curve $V_{r}=f(X)$ is a lack what reduces metrological importance of this effect. But this lack can be easily eliminated by application of calibration.

\section{References}

1. M. Frokht, Photoelastisity (2 volumes) Vol. 1. OGIZ, Moscow, 1948 (in Russian).

2. M. Born, E. Wolf, Principles of optics. Nauka, Moscow, 1970 (in Russian).

3. V.V. Mar'enko, B.H. Kolesnichenko, S.H. Savenkov, The automated system for research of polarized structure of an optical field, scattered by the natural objects // Optics of atmosphere and ocean 6, p. 1460-1464 (1993) (in Russian).

4. S.N. Jasperson, S.E. Sahnatterly, An improved method for high reflectivity ellipsometry based on a new polarization modulation technique // Rev. Sci. Instr. 40 p. $761-767$ (1969).

5. B.K. Serdega, Ye.F. Venger, Ye.V. Nikitenko, The thermoelasticity in Ge due to nonuniform distribution of doping impurity studied by light polarization modulation technique // Semiconductor Physics, Quantum Electronics \& Optoelectronics 2, p. 153-156 (1999).
6. Ye.V. Nikitenko, B.K. Serdega, Features of use of the polarization modulator in the optical schema for research birefringence // Optoelectronics and Semiconductor Technics (Naukova Dumka, Kyiv) No 33, p. 102-108 (1998) (in Russian).

7. D.E. Aspnes, J.P. Harbison, A.A. Studna, and L.T. Florez, Application of reflectance difference spectroscopy to molecular-beam epitaxy growth of GaAs and AlAs // J. Vac. Sci. Technol. A6, p. 1327-1332 (1988).

8. A. Jerard, J.M. Berch, Introduction in matrix optics. Mir, Moscow, 1978 (in Russian).

9. J. Schmidt-Tiedemann, Experimental evidence of birefringence by free carriers in semiconductors // Phys. Rev. Lett. 7, p. 372-374 (1961).

10. W.C. Dash, F. Newman, Intrinsic optical absorption single-crystal $\mathrm{Ge}$ and $\mathrm{Si}$ at 77 and $300 \mathrm{~K} / /$ Phys. Rev. 99, p. 1151-1155 (1955).

11. V.I. Nikitenko, G.P. Marninenko, Some photoelastic properties of gallium arsenide and silicon // Fizika Tverd. Tela 7, p. 622-624 (1965) (in Russian).

12. M. Chandrasekhar, M.H. Grimsditch, M. Cardona, Piezobirefringence above the fundamental edge in Si // Phys. Rev. B18, p. $4301-4310$ (1978). 